\title{
MOMENTS OF THE LIFETIME OF CONDITIONED BROWNIAN MOTION IN CONES
}

\author{
BURGESS DAVIS AND BIAO ZHANG
}

(Communicated by Lawrence F. Gray)

\begin{abstract}
Let $\tau$ be the time it takes standard $d$-dimensional Brownian motion, started at a point inside a cone $\Gamma$ in $\mathbb{R}^{d}$ which has aperture angle $\theta$, to leave the cone. Burkholder has determined the smallest $p$, denoted $p(\theta, d)$, such that $E \tau^{p}=\infty$. We show that if $y \in \partial \Gamma$ then the smallest $p$, such that $E\left(\tau^{p} \mid B_{\tau}=y\right)=\infty$, is $p=2 p(\theta, d)+(d-2) / 2$.
\end{abstract}

We will be working with spherical coordinates in $\mathbb{R}^{d}, d \geq 2$. Let, for a point $x=\left(x_{1}, \ldots, x_{d}\right) \in \mathbb{R}^{d},|x|=\left(\sum x_{i}^{2}\right)^{1 / 2}$, and let $\varphi$ be the angle that the line segment connecting the origin 0 and $x$ makes with the line segment connecting the origin and $1=(0,0, \ldots, 0,1)$. Let, for $0<\theta<\pi, \Gamma=\Gamma(d, \theta)$ be the cone $\{\varphi<\theta\}$. We use $\tau_{D}$ to designate the exit time of a process from a domain $D$, and we shorten $\tau_{\Gamma}$ to $\tau$. Probability and expectation for standard $d$-dimensional Brownian motion started at $x$ will be denoted by $P_{x}$ and $E_{x}$, and if $y \in \partial \Gamma$ (boundary of $\Gamma$ ), $P_{x}^{y}$ and $E_{x}^{y}$ designate probability and expectation for this motion conditioned to exit $\Gamma$ at $y$ or, more formally, of the $h$-process, with $h$ the Poisson kernel of $\Gamma$ for the boundary point $y$. We will discuss $h$-processes in more detail later.

Let $p(\theta, 2)=\pi / 2 \theta$, and, for $d>2$, put $p(\theta, d)=2 \sup \left\{x: \theta<\lambda_{x, d}\right\}$, where $\lambda_{x, d}$ is the smallest positive zero of the hypergeometric function

$$
h(w)=F(-x, x+d-2,(d-1) / 2 ;(1-\cos w) / 2),
$$

with $F(a, b, c ; t)=\sum_{k=0}^{\infty} \frac{(a)_{k}(b)_{k} k^{k}}{(c)_{k} !}$, and $(r)_{k}=r(r+1) \cdots(r+k)$. In [1] it is shown that for $x \in \Gamma$ and $p>0, E_{x} \tau^{p}<\infty$ if and only if $p<p(\theta, d)$. This was sharpened and generalized by [4]. Our main result is

Theorem 1. Let $x \in \Gamma, y \in \partial \Gamma$, and $p>0$. Then $E_{x}^{y} \tau^{p}<\infty$ if and only if $p<2 p(\theta, d)+(d-2) / 2$.

Proof. Our proof of this theorem essentially involves giving a new proof of Burkholder's result which, with little alteration, can be used for conditioned Brownian motion, although we note that this "new" proof rests on a calculation originally made by Burkholder. Let $\Gamma_{n}=\Gamma \cap\left\{|x| \leq 2^{n}\right\}, S_{n}=\Gamma \cap\left\{|x|=2^{n}\right\}$,

Received by the editors May 18, 1992 and, in revised form, October 26, 1992.

1991 Mathematics Subject Classification. Primary 60J65, $60 \mathrm{~J} 05$.

Key words and phrases. Conditioned Brownian motion, $h$-processes.

This research was supported by a National Science Foundation Grant. 
and $H_{n}=S_{n} \cap\{\varphi \leq \theta / 2\}$ be the middle half of $S_{n}$. We first prove Theorem 1 in the case $x=1$ and $y=0$, and then explain how to extend the proof to the general case. Let $\tau_{n}$ be the first time a process hits, $S_{n}$. Then

$$
E_{1} \tau^{p}=\sum_{n=0}^{\infty} E_{1}\left(\tau^{p} \mid \tau_{n}<\tau \leq \tau_{n+1}\right) P_{1}\left(\tau_{n}<\tau \leq \tau_{n+1}\right)
$$

and

$$
E_{1}^{0} \tau^{p}=\sum_{n=0}^{\infty} E_{1}^{0}\left(\tau^{p} \mid \tau_{n}<\tau \leq \tau_{n+1}\right) P_{1}^{0}\left(\tau_{n}<\tau \leq \tau_{n+1}\right) .
$$

We will show

$$
E_{1}\left(\tau^{p} \mid \tau_{n}<\tau \leq \tau_{n+1}\right) \sim E_{1}^{0}\left(\tau^{p} \mid \tau_{n}<\tau \leq \tau_{n+1}\right) \sim 2^{2 n p},
$$

where $a_{n} \sim b_{n}$ means that $a_{n} / b_{n}$ is bounded above and below by positive constants which, while they may depend on $\theta, d$, and $p$, do not depend on $n \geq 0$. We also show that there is an $\alpha=\alpha(\theta)>0$ such that both of the following hold:

$$
\begin{gathered}
P_{1}\left(\tau_{n}<\tau \leq \tau_{n+1}\right) \sim 2^{-n \alpha} . \\
P_{1}^{0}\left(\tau_{n}<\tau \leq \tau_{n+1}\right) \sim 2^{-n[2 \alpha+d-2]} .
\end{gathered}
$$

Relationships (1)-(5) imply $E_{1} \tau^{p}=\infty$ if and only if $\sum_{n=1}^{\infty} 2^{2 n p} 2^{-n \alpha}=\infty$ and $E_{1}^{0} \tau^{p}=\infty$ if and only if $\sum_{n=1}^{\infty} 2^{2 n p} 2^{-n[2 \alpha+d-2]}=\infty$. Thus $E_{1} \tau^{p}=\infty$ if and only if $p \geq \alpha / 2$, which with Burkholder's result gives $p(d, \theta)=\alpha / 2$, while $E_{1}^{0} \tau^{p}=\infty$ if and only if $p \geq[2 \alpha+d-2] / 2=2 p(d, \theta)+(d-2) / 2$, verifying Theorem 1 in the special case $x=1, y=0$.

To complete the proof of Theorem 1 in this special case we need to prove (3)(5). Before we do, we collect some of the tools we will use. We let $P_{x}^{h, D}=P_{x}^{h}$ and $E_{x}^{h, D}=E_{x}^{h}$ denote probability and expectation for the $h$-process in a domain $D$ with associated harmonic function $h$. Here, the only $h$-processes we will be concerned with are Brownian motion conditioned to exit a domain at a specified point or set. For a formal description of $h$-processes and proofs of the properties of $h$-processes stated below, see [5]. Let $G$ be a subdomain of $D, x \in G, h$ harmonic in $D$. Then the exit distribution from $G$ under $P_{x}^{h}$ is given by

$$
P_{x}^{h}\left(B_{\tau_{G}} \in A\right)=\int_{A} \frac{h(z)}{h(x)} d P_{x}\left(B_{\tau_{G}}=z\right), \quad A \subset \partial G, A \text { Borel } .
$$

Furthermore, conditioned on $B_{\tau_{G}}$, the process $B_{t}, 0 \leq t \leq \tau_{G}$, has the same distribution under both $P_{x}$ and $P_{x}^{h}$. Especially, the distribution of the exit time of $B_{t}$ from the open ball $B(x, \delta) \subset D$, of center $x$ and radius $\delta$, is the same under both $P_{x}$ and $P_{x}^{h}$, since this distribution conditioned on the exit position from the ball is the same and by symmetry does not depend on the exit position, under $P_{x}$.

In the following inequalities, $c, C, C_{p}$, etc., stand for generic positive constants, which may depend on $\theta$ and $d$ but do not depend on $n$. Let the harmonic functions $u$ and $v$ be defined in $\Gamma_{1}$ by $u(x)=P_{x}\left(B_{\tau_{\Gamma_{1}}} \in S_{1}\right)$ and $v(x)=P_{x}\left(B_{\tau_{\Gamma_{1}}} \in H_{1}\right)$. 
Lemma 1. If $x \in \Gamma_{1}$ and $|x| \leq 1$, then $u(x)<C v(x)$.

Proof. A direct probabilistic proof is not too difficult, but since Lemma 1 follows immediately from the boundary Harnack principle for Lipschitz domains (see [7]), we take this route. This principle implies that, given $x \in \partial \Gamma \cup\{|x| \leq$ $1\}$, there is a $\delta(x)>0$, such that $u(y)<C v(y)$ if $y \in \Gamma \cap B(x, \delta(x))$. Since we can pick a finite number of $x$ such that the union of the $B(x, \delta(x))$ for these $x$ contains $\{\partial \Gamma\} \cap\{|x| \leq 1\}$ and since clearly $u(y)<C v(y)$ for $y$ in a compact subset of $\Gamma_{1}$, Lemma 1 follows.

Now let $K(x)$ be the Poisson kernel for $\Gamma$ with respect to the point 0 ; that is, $K$ is the unique function in $\Gamma$ which is harmonic and positive, has limit zero as either $\infty$ or a nonzero boundary point is approached, and satisfies (is normalized so that) $K(1)=1$. Scaling shows there is a positive number $\beta$ and a positive function $g$ on $[0, \theta)$ such that $K(x)=g(\varphi) /|x|^{\beta}$. The exponent $\beta=\beta(\theta)>0$ was found in [1]. We also note that $M(x)=K\left(x /|x|^{2}\right) /|x|^{d-2}=$ $|x|^{\beta+2-d} g(\varphi)$ is harmonic in $\Gamma$ (see [6, p. 36]). Let $\alpha=\beta+2-d$, so $M(x)=$ $|x|^{\alpha} g(\varphi)$.

Lemma 2. For each $p>0$ there is a constant $C_{p}$ such that if $h$ is harmonic in $\Gamma_{1}$ and $x \in \Gamma_{1}$,

$$
E_{x}^{h} \tau^{p}<C_{p} .
$$

Proof. That $\sup _{x, h} E_{x}^{h} \tau<\infty$ is a result of Cranston [2], and the argument that extends this to (7) is standard (see the end of the first section in [3]).

Now we prove (3)-(5), starting with (4). Note that $\lambda=\max \{g(\varphi): \varphi<$ $\theta\}<\infty$ and $\eta=\min \{g(\varphi): \varphi \leq \theta / 2\}>0$. The fact that $1=M(1)=$ $E M\left(B_{\tau_{n}}\right)=E M\left(B_{\tau_{n}}\right) I\left(\tau_{n}<\tau\right)$, where $I$ denotes indicator function, together with Lemma 1 and scaling, gives $c P_{1}\left(\tau_{n}<\tau\right)\left(2^{n}\right)^{\alpha}<1<C P_{1}\left(\tau_{n}<\tau\right)\left(2^{n}\right)^{\alpha}$. Clearly $P_{x}\left(\tau_{n+1}>\tau\right)>c, x \in S_{n}$, and this, together with the preceding inequalities, gives (4).

Next we prove (5). We have, by (6) with $h=K$, recalling that $g(1)=$ $K(1)=1$,

$$
P_{1}^{0}\left(B_{\tau_{\Gamma_{n}}} \in S_{n}\right) \leq \lambda\left(2^{-n}\right)^{\beta} P_{1}\left(B_{\tau_{\Gamma_{n}}} \in S_{n}\right) \leq C \lambda 2^{-n \beta} 2^{-n \alpha},
$$

where the last inequality follows from (4). Furthermore, again by (6) in the second inequality and Lemma 1 in the third,

$$
\begin{aligned}
P_{1}^{0}\left(B_{\tau_{\Gamma_{n}}} \in S_{n}\right) & \geq P_{1}^{0}\left(B_{\tau_{\tau_{n}}} \in H_{n}\right) \geq \eta P_{1}\left(B_{\tau_{\tau_{n}}} \in H_{n}\right)\left(2^{-n}\right)^{\beta} \\
& \geq c P_{1}\left(B_{\tau_{\tau_{n}}} \in S_{n}\right) 2^{-n \beta} \geq c 2^{-n \beta} 2^{-n \alpha} .
\end{aligned}
$$

Together with (8), this proves $(5)$.

Next we prove (3). Let $G_{n}=\left\{\tau_{n}<\tau \leq \tau_{n+1}\right\}$. On $G_{n}, \tau=\tau_{n}+\left(\tau-\tau_{n}\right)$. That $E_{1}\left(\tau_{n}^{p} \mid \tau_{n}<\tau\right)<C_{p} 2^{2 n p}$ follows from Lemma 2, with $h=u$ and scaling, and since $P_{x}\left(\tau_{n+1}>\tau\right)>c, x \in S_{n}$, we have $P_{x}\left(G_{n}\right)>c P_{x}\left(\tau_{n}<\tau\right)$. Thus

$$
E_{1}\left(\tau_{n}^{p} \mid G_{n}\right)<C_{p} 2^{2 n p} .
$$

The inequality

$$
E_{1}^{0}\left(\tau_{n}^{p} \mid \tau_{n}<\tau\right)<C_{p} 2^{2 n p}
$$


follows from Lemma 2 with $h=u$ and scaling, recalling the first sentence after inequality (6). Now

$$
P_{x}^{0}\left(\tau_{n+1}>\tau\right)>c, \quad x \in H_{n},
$$

since $P_{x}^{0}\left(\tau_{n+1}>\tau\right)$ is a positive continuous function on $H_{n}$. That $c$ may be chosen independently of $n$ in (11) follows from scaling. Since

$$
P_{1}^{0}\left(B_{\tau_{n}} \in H_{n}\right)>c P_{1}^{0}\left(B_{\tau_{n}} \in S_{n}\right),
$$

by Lemma 1 and formula (6), we have from (11) that $P_{1}^{0}\left(G_{n}\right)>c P_{1}^{0}\left(\tau_{n}<\tau\right)$, and this together with (10), gives

$$
E_{1}^{0}\left(\tau_{n}^{p} \mid G_{n}\right)<C_{p} 2^{2 n p} .
$$

The inequalities

$$
E_{1}^{0}\left(\left(\tau-\tau_{n}\right)^{p} \mid G_{n}\right)<C_{p} 2^{2 n p}
$$

and

$$
E_{1}\left(\left(\tau-\tau_{n}\right)^{p} \mid G_{n}\right)<C_{p} 2^{2 n p}
$$

follow by very similar reasoning. We just prove (14). Now

$$
\begin{aligned}
E_{1}^{0}\left(\tau-\tau_{n}\right)^{p} I\left(G_{n}\right) & =E_{1}^{0} E_{1}^{0}\left[\left(\tau-\tau_{n}\right)^{p} I\left(G_{n}\right) \mid B_{\tau_{n}}\right] \\
& =E_{1}^{0} E_{B_{\tau_{n}}}^{0}\left(\tau^{p} I\left(\tau<\tau_{n+1}\right)\right) I\left(\tau_{n}<\tau\right) \\
& =E_{1}^{0} E_{B_{\tau_{n}}}^{\xi, \Gamma_{n+1}} \tau^{p} P_{B_{\tau_{n}}}^{0}\left(\tau<\tau_{n+1}\right) I\left(\tau_{n}<\tau\right) \\
& \leq E_{1}^{0} C_{p} 2^{2(n+1) p} P_{B_{\tau_{n}}}^{0}\left(\tau<\tau_{n+1}\right) I\left(\tau_{n}<\tau\right) \\
& =C_{p} 2^{2(n+1) p} P_{1}^{0}\left(G_{n}\right),
\end{aligned}
$$

where the function $\xi$ is the Poisson kernel for the point 0 for the domain $\Gamma_{n+1}$, and the inequality follows from Lemma 2 and scaling. We use (6) and the sentence after (6) to justify the third equality.

Now if $\lambda$ is the distance of $H_{1}$ from $\partial \Gamma$, then $2^{n} \lambda$ is the distance from $H_{n}$ to $\partial \Gamma$, and if $v_{n}=\inf \left\{t:\left|B_{t}-x\right|=2^{n} \lambda\right\}$, we have, as in (16),

$$
\begin{aligned}
E_{1}^{0} \tau^{p} I\left(G_{n}\right) & \geq E_{1}^{0}\left(\tau-\tau_{n}\right)^{p} I\left(G_{n}\right) \\
& =E_{1}^{0} E_{B_{n}}^{\xi, \Gamma_{n+1}} \tau^{p} P_{B_{\tau_{n}}}^{0}\left(\tau<\tau_{n+1}\right) I\left(\tau_{n}<\tau\right) \\
& \geq E_{1}^{0} E_{B_{\tau_{n}}, \Gamma_{n+1}}^{\xi} v_{n}^{p} P_{B_{\tau_{n}}}^{0}\left(\tau<\tau_{n+1}\right) I\left(\tau_{n}<\tau, B_{\tau_{n}} \in H_{n}\right) \\
& =E_{1}^{0} C_{p} 2^{2 n p} P_{B_{\tau_{n}}}^{0}\left(\tau<\tau_{n+1}\right) I\left(\tau_{n}<\tau, B_{\tau_{n}} \in H_{n}\right) \\
& =C_{p} 2^{2 n p} P_{1}^{0}\left(G_{n}, B_{\tau_{n}} \in H_{n}\right)>C_{p} 2^{2 n p} P_{1}^{0}\left(G_{n}\right),
\end{aligned}
$$

where we recall the second sentence after (6), and use scaling to obtain the next to the last inequality, and use (11) and (12) to prove the last inequality. Rephrased, this becomes

$$
E_{1}^{0}\left(\tau^{p} \mid G_{n}\right)>C_{p} 2^{2 n p},
$$

and similarly we can prove

$$
E_{1}\left(\tau^{p} \mid G_{n}\right)>C_{p} 2^{2 n p} .
$$


Together, (9), (13)-(15), (17), and (18) establish (3), and thus Theorem 1, in the special case that $x=1$ and $y=0$, is proved.

Finally, we prove the general case. For $y \in \partial \Gamma$, that $E_{x}^{y} \tau^{p}$ is either finite for all $x \in \Gamma$ or infinite for all $x \in \Gamma$, follows from the well-known argument that shows the analogous result for $E_{x} \tau^{p}$, which we will not repeat. Since, if $a$ is positive, the distribution of $\tau$ under $P_{a x}^{a y}$ is the distribution of $a^{2} \tau$ under $P_{x}^{y}$, evidently $E_{x}^{y} \tau^{p}$ is either finite for all $x \in \Gamma$ and nonzero $y \in \partial \Gamma$ or infinite for all these $x, y$. To finish the proof, it suffices to show that there is just one $y \neq 0, y \in \partial \Gamma$, such that for all $p, E_{1}^{0} \tau^{p}$ and $E_{1}^{y} \tau^{p}$ are finite for exactly the same values of $p$. Pick $y$ such that $|y| \leq \frac{1}{2}$. Let $K^{\prime}$ be the Poisson kernel for $\Gamma$ for the point $y$, normalized so that $K^{\prime}(1)=1$. Now it follows easily from Theorem 5.20 of Jerison and Kenig (1982) that $c K(x)<K^{\prime}(x)<C K(x)$, $x \in \Gamma,|x| \geq 1$, and thus the proof of the $E_{1}^{0}$ case of Theorem 1 works essentially without change, to show that $E_{1}^{y} \tau^{p}$ is finite for the same $p$ for which $E_{1}^{0} \tau^{p}$ is finite. This finishes our proof of Theorem 1 .

\section{REFERENCES}

1. D. L. Burkholder, Exit times of Brownian motion, harmonic majorization, and Hardy spaces, Adv. Math. 26 (1977), 182-205.

2. M. Cranston, Lifetime of conditioned Brownian motion in Lipschitz domains, Z. Wahrsch. Verw. Gebiete 70 (1985), 335-340.

3. B. Davis, Conditioned Brownian motion in planar domains, Duke Math. J. 57 (1988), 397-421.

4. R. D. DeBlassie, Exit times from cones in $\mathbb{R}^{n}$ of Brownian motion, Probab. Theory Related Fields 74 (1987), 1-29.

5. J. L. Doob, Classical potential theory and its probabilistic counterpart, Springer, New York, 1984.

6. L. L. Helms, Introduction to potential theory, Wiley, New York, 1969.

7. D. S. Jerison and C. E. Kenig, Boundary value problems on Lipschitz domain, Partial Differential Equations (Walter Littman, ed.), MAA Stud. Math., vol. 23, Math. Assoc. Amer., Washington, DC, 1982, pp. 1-68.

Department of Mathematics, Purdue University, West Lafayette, Indiana 47907-1399 E-mail address, B. Davis: bdavis@stat.purdue.edu

E-mail address, B. Zhang: Ibz@math.purdue.edu 